\title{
Festschrift on the occasion of Ulrike Feudel's 60th birthday
}

\author{
Jan A. Freund ${ }^{1, a}$, Ksenia Guseva ${ }^{1}$, and Celso Grebogi ${ }^{2}$ \\ 1 Institute for Chemistry and Biology of the Marine Environment, University of \\ Oldenburg, Carl-von-Ossietzky-Str. 9-11, 26129 Oldenburg, Germany \\ 2 Institute for Complex Systems and Mathematical Biology, King's College, University of \\ Aberdeen, Old Aberdeen AB24 3UE, UK
}

Received 16 May 2017

Published online 21 June 2017

Since the early days of chaos theory $[1,2]$ and complexity research [3], and the boom of results obtained since the 1980s, we have witnessed a growing body of work on the applications of nonlinear dynamics, chaos and complexity in diverse fields of science, e.g., in neuronal dynamics and brain research, or laser dynamics and excitable media in chemistry, or the dynamics of ecological populations and communities, to mention but a few. On the one hand, these applications fueled a revived interest in synchronization phenomena and control of networks. On the other hand, they inspired investigations of the effects of the coupling topology in networks where each node represents a subsystem with an intrinsic nonlinear dynamics; between the classical extremes of next neighbor coupling (diffusive) and the global all-to-all coupling (mean field) interesting new phenomena can be found such as, for instance, chimera states that have come into the focus of research recently. While chaos is often defined and observed in deterministic nonlinear dynamics, the importance of temporal fluctuations and noise is widely acknowledged and, in particular, the cooperative action of noise and nonlinearities can enrich the repertoire of dynamical phenomena and the emergence of complex structures.

The European Physics Journal Special Topics (EPJST) edition at hand collects a series of articles reflecting recent advances in nonlinear dynamics and complex structures. Contributors to this issue are renowned specialists in a field belonging to nonlinear research and, in fact, some of the authors even established its foundations. While some contributions of this issue still tackle fundamental aspects of nonlinear dynamics, the majority of articles in this collection uses the arsenal of nonlinear methods to model questions belonging to a topical field. We tried to map this partition by dividing the present edition into sections.

A first section on Fundamental Aspects of Nonlinear Dynamics is opened with a mini-review by Lai and Grebogi [4] whose main purpose is to argue that quasiperiodicity tends to suppress multistability, as does random noise. Through an analysis of

\footnotetext{
${ }^{a}$ e-mail: jan.freund@uni-oldenburg.de
} 
quasiperiodically driven systems (a damped pendulum and a semiconductor superlattice), they show that quasiperiodic driving indeed can eliminate multistability and generate robust chaos. Robust chaos, i.e., the persistence of a chaotic attractor when control parameters are varied, is also in the focus of the review by Glendinning [5] who discusses different approaches to robust chaos and dwells on the extent to which the mechanisms of its generation are understood. As an outcome he provides a refinement of Young's theorem and verifies its conditions numerically. The article by Rankin and Osinga [6] touches on the properties of periodic channels, i.e., multi-dimensional generalizations of periodic windows in a one-parameter bifurcation diagram, that occur in a locus of boundary crisis. By varying parameters of the Ikeda map the authors reconstruct geometric properties of periodic channels and neighboring chaotic attractors. Similar observations were reported for a three-parameter study of a quasiperiodically forced Hénon map, but are novel for two-dimensional maps. The article by Saiki et al. [7] draws a connection to the classical Lorenz equations by considering their embedding in a whole family of related differential equations. Restricting to a particular subclass for which trajectories stay on the three-sphere they find a surprisingly rich variety of dynamics hitherto unknown for Lorenz systems. Strange nonchaotic attractors (SNAs) are addressed by the article of Doroshenko and Kuznetsov [8]. By mapping a specific system of ODEs to its corresponding Hunt and Ott map they can investigate a robust SNA system. Numerical results indicate that a SNA indeed is found and the authors confirm this finding by analyzing the topological features of the map for related phase variables. A system exhibiting transient chaos is in the focus of the contribution by Felk et al. [9]. Here, transient chaos is constructed by two coupled twist maps with weak dissipation and is associated with a chaotic saddle situated near the resonance web of the Hamiltonian system and that gets destroyed with increasing dissipation. Chaos control by feedback is now well-known. In the paper by Arecchi et al. [10] an alternative scheme is discussed, the so-called phase control of chaos. In their contribution the authors investigate the application of square pulses to the paradigmatic driven Duffing oscillator with a focus on the question of where to apply the control term to reach the best efficiency and how effectiveness depends on the details of the duty cycle. The first section is completed by a research paper contributed by Politi et al. [11] in which the authors show if and how collective chaos, defined as irregular behavior on macroscopic scales, can emerge in mean-field coupled identical phase-oscillators. In the context of identical units they introduce a new way of representing the microscopic configurations which allows for a natural comparison between the microscopic and macroscopic regimes in terms of the range of finite perturbations. Most interestingly, this leads to the detection of a mesoscopic range where neither the microscopic nor the macroscopic rule applies.

The second section encompasses articles related to the topics of Network Dynamics and Chimera States. Hellen et al. [12] map genetic networks to electronic circuits with improved design which allows them to study the former through numerical simulations and mathematical analyses with the aim of precisely controlling the initial conditions when studying a multistable system such as repressilators coupled by quorum-sensing. In this way they find coexistence of in-phase and anti-phase limit cycles, noise-induced transitions between these states, and the influence of high period limit cycles on the complex dynamics. In [13] Rubido et al. present general solutions for current conservative DC/AC circuit networks with resistive, capacitive, and/or inductive edge characteristics, proposing an alternative to Kirchhoff's equations that is advantageous for constantly changing locations of inputs and outputs. Their novel approach provides a rigorous link between network topology and the steady-state currents of a conservative circuit network. An application of network dynamics on the global scale is shared by Brenner et al. [14] who use a complex network approach to model and analyze a disease spreading dynamics. The SEIR 
(susceptible-exposed-infected-recovered) compartmental model they use is indeed inspired by real world scenarios and via modulating the transmission rate by climatic factors they focus on aspects of climate change. In this way they find that spreading patterns of infectious diseases depend on both the network structure and the climatic setup of the environment. The next three articles are devoted to the so-called chimera states, i.e., the robust coexistence of coherence and incoherence in networks of identical oscillators with non-local coupling. The contribution by Rybalova et al. [15] is inspired by the question of how the properties of individual ensemble elements can influence the appearance of chimera states. To solve this question the authors study coherence-incoherence transitions in ensembles of non-locally coupled Hénon and Lozi maps, describe their similarities and fundamental differences and find that completely synchronized chaos exists in both systems for dominant coupling, as does desynchronized spatio-temporal chaos for weak coupling. In [16] Maistrenko et al. study the effects of non-local coupling in three-dimensional networks of Kuramoto phase oscillators and investigate conditions for the emergence and stability of scroll wave chimeras. As a result of their demanding numerical simulations they find complex chimera states in three dimensions (Hopf links and trefoils) that exist only for nonlocal coupling and sufficiently large phase shift between oscillators. The final paper in the second section by Sawicki et al. [17] reveals how the interplay of complex network topology and time delay influences chimera states and other complex spatio-temporal patterns. Studying a ring of $N$ identical van der Pol oscillators with different coupling topologies the authors find that the interplay of a complex hierarchical network topology and time delay results in a plethora of patterns beyond those observed in regular two-population or non-locally coupled ring networks. They thus provide evidence that time delay can play a key role for promotion or destruction of chimera patterns.

The third section contains four articles under the section title Synchronization. The contribution by Banerjee et al. [18] explores the constructive role of heterogeneity for synchronization in chaotic Rössler oscillators using different coupling configurations (a one-dimensional open array, a star network, a ring of oscillators and a two-dimensional lattice of oscillators). Through numerical simulations they show that synchronization can be enhanced by an induced heterogeneity or a parameter mismatch in a suitably located central node that works as a relaying device. The combined effects of conjugate coupling and time-delay in nonlinear oscillators are reported in the paper by Sharma et al. [19]; this setup is interesting, as a coupling of dissimilar variables on its own already mimics time delay. For a paradigmatic limit cycle oscillator (StuartLandau) and an ecological model (enlarged Truscott-Brindley), and with coupling implemented through cross diffusion terms, they find that this coupling scheme can induce an anomalous transition from amplitude or oscillation death to a state of desynchronized motion, even for identical oscillators. Synchronization in network motifs of delay-coupled neurons is at the heart of an analysis contributed by Sausedo-Solorio and Pisarchik [20]. Through simulations of Rulkov neurons (twodimensional nonlinear maps) the authors analyze how network motifs synchronize in the presence of a synaptic delay and a feedback loop and in particular how synchronization depends on the coupling strength and synaptic delay. The fourth paper in this section by Goldobin et al. [21] provides a detailed study of competition between common noise and a desynchronizing global coupling of the Kuramoto-Sakaguchi type. The basic model is an ensemble of infinitely many phases and allows for a comprehensive analytical treatment via Watanabe-Strogatz and Ott-Antonsen approaches. Most interesting effects appear when the noise and the coupling compete.

A fourth section collects articles that belong to the fields of Neuronal Dynamics and Extreme Events in Excitable Systems. In [22] Shaffer et al. investigate a tonic (rhythmic single spiking) to bursting (repeating sequences of multiple spikes) 
transition that occurs in a linear chain of three synchronous model neurons (called triads) connected via electrical synapses. To this end they perform numerical simulations of three Huber-Braun model neurons, mimicking electrical synapses through additional ionic currents, and show striking differences between the bifurcation scenarios in single neurons and in coupled triads. Khamesian and Neiman, in their contribution [23], study the noisy dynamics of a simple nonlinear model for the hair bundle of the bullfrog saccular hair cell. Control parameters used in this study are the strength of fast adaptation and the membrane potential. Their results suggest that fast adaptation significantly affects the dynamics of the hair bundle. A description of neuronal networks in terms of scale-free networks of excitable systems (FitzHughNagumo units) is underlying the article shared by Rings et al. [24] in which the authors aim to clarify the role of high- and low-degree nodes in the generation of extreme events. Through their analysis they find that low-degree nodes trigger extremes via so-called proto-events while hubs propagate these to the initially inactive nodes. Extreme events are also investigated in the contribution by Martinez Alvarez et al. [25], however, now with a focus on identifying early-warning signals of extreme events. Employing a method of ordinal statistics to pronounced peaks of a time series, generated by a model of laser dynamics, they are able to detect ordinal patterns preceding extreme events. Being a proof of concept, the proposed method may be useful for other systems where extremes are generated by a mechanism that is dominated by a deterministic dynamics.

A fifth section collects research papers that may be titled Fluctuations and Noise in Complex Systems. In [26] Sprott et al. investigate a periodically forced chaotic system similar to the forced van der Pol oscillator but with a spatially-periodic damping (cosine term). What they find is a system with an infinite number of coexisting attractors, including limit cycles, attracting tori, and strange attractors, something they call megastability. A periodical forcing of the Brusselator in the low-frequency limit of the drive is investigated by Freire et al. [27]. A remarkable finding is that while this low-frequency limit is largely free of chaos it contains a dense net of oscillatory solutions whose waveforms comprise a number of spikes growing without bound as a function of the control parameters. Electrochemical oscillations on nanoscale electrodes are considered by Cosi and Krischer [28]. Of special interest is a comparison with chemical oscillations in small systems where molecular noise becomes important. Performing simulations of a prototypical potentiostatic oscillator that exhibits negative differential resistance, they demonstrate peculiar features of electrochemical nanoscale oscillators that are linked to molecular noise. The role of nonlinearities for the conversion of chemical into other forms of energy (electrical or ecological) is investigated in a contribution by Ebeling and Feistel [29]. They aim at examples with high efficiency and, in this context, transfer the SET (Schweitzer-Ebeling-Tilch) depot model to a fuel cell and to a predator prey dynamics. They find that high efficiency needs the exploitation of nonlinear effects and an optimization. In [30] Drótos et al. are concerned with climate dynamics and its strong internal variability. In the framework of a dynamical systems approach this suggests the notion of a chaotic attractor and in combination with climate change leads to the concept of a snapshot attractor, naturally arising in an ensemble description. Using an intermediate-complexity general circulation model (the Planet Simulator) they argue that it is important to check whether convergence to the attractor is reached. The final article in this fifth section by Milster et al. [31] presents a consistent procedure of adiabatic elimination of the orientational variable of a micro-swimmer which is an active Brownian particle moving at constant speed but with fluctuating orientation. Equations resulting via systematic elimination procedures, starting either from the Langevin equation or the related Fokker-Planck equation, still reflect important microscopic properties of the micro-swimmer such as, for instance, the conservation of kinetic energy. Only 
after averaging over random orientations does the particle lose this property, and the description becomes similar to that of a normal overdamped Brownian particle.

In a sixth section we group four contributions that investigate Dynamics in Flows. In [32] Ser-Giacomi et al. elaborate on Lagrangian Flow Networks (LFNs). These arise in coarse-grained descriptions of a fluid dynamics where nodes correspond to small regions in the fluid domain and mass transfer between these regions defines weighted links. Based on this method, some properties of open flows such as, for instance, escape rates are mapped to network measures. The authors illustrate this with a simple model system and confirm that the LFN approach is useful even for a description of open flows. Describing the advection of small inertial particles in different flow types naturally leads to the concept of the history force. In their contribution Guseva et al. [33] investigate the appearance of long transients in the presence of the history force. Using the so-called snapshot attractor approach enables exploitation of a time-scale separation, thus allowing the authors identifying a short-term exponential convergence and a long-term power law behavior. A description of the transport of inertial particles is also what Vilela and Oliveira [34] are concerned with. For inertial particles denser than the carrying flow (aerosols) the authors propose an effective description of the particle advection dynamics by the so-called Stokes map. The authors dwell on the qualitative similarities between the dynamics given by the Maxey-Riley equation and the Stokes map. The last article in the section on fluid dynamics is a paper by Dan et al. [35] who investigate the bursting dynamics in a Rayleigh-Bénard convection model when the Rayleigh-number is modulated slowly but periodically near its instabilities or the bifurcation points. Applying limits to the Boussinesq equations and performing bifurcation analyses the authors show that the quasi-static dynamical system exhibits a rich bifurcation pattern close to the onset of Rayleigh-Bénard convection in low Prandtl number fluids.

The final seventh section comprises contributions that belong to the field of Ecological Dynamics. In [36] van Voorn and Kooi advocate a combination of bifurcation and sensitivity analyses in the study of ODE models. They exemplify their argument by applying the proposed approach to three representations of a predator-prey model thus showing its usefulness for model development and analysis. A sensitivity analysis is used by Chakraborty et al. [37] to study the role of agricultural impact on the formation of harmful algal blooms. Based on a six-component ODE model, and through a qualitative analysis and numerical simulations they investigate the role of a fertilizer input rate on different dynamical features of the system, thus tracing anthropogenic activities in the occurrence of algal blooms and dissolved oxygen levels. A better understanding of the role of motifs and their dynamical features in food web networks surely is beneficial and desirable. Karnatak and Wollrab [38] explore the dynamical consequences of a shift between two central tritrophic food web motifs, namely an intraguild predation (trophic interaction between natural antagonists) motif and a food chain. They draw interesting ecological conclusions from analyzing a dynamical flow system in which they smoothly alter the interaction topology. It is widely recognized that space is an essential dimension for many ecological phenomena. Interacting ecological populations distributed in space are addressed by Arumugam et al. [39] with the objective of clarifying the effect of species dispersal on a spatial ecological system consisting of heterogeneous fragmented habitats. Using a so-called meta-population model, in which fragmented habitats are connected by mean-field diffusive coupling, they find that dispersal enhances rhythmic oscillations and the synchrony-stability relationship. A spatial aspect is also behind the biological invasion modeled by Siekman et al. [40] using a spatio-temporal Lotka-Volterra model with non-standard nonlinear noise (saturating environmental noise intensity mimicking that individuals do not respond independently to environmental fluctuations). They examine in more detail the combined effect of Fokker-Planck diffusion and nonlinear noise and arrive 
at predictions different from those of standard models for environmental fluctuations. The final article of this section - and of this EPJST issue - is a report by Zelnik and Tzuk [41] who focus on wavelength selection beyond the Turing regime (where a uniform state is unstable to small non-uniform perturbations) in models of dryland ecosystems. They step beyond the Turing regime by moving into the bistable region and by considering the effect of disturbances that are not locally small and, therefore, beyond a linear approximation. Using simulations from three different models of dryland vegetation and analyzing them they derive a relation between the wavelength of the highest growth mode and the so-called snaking wavelength.

This broad overview makes clear that elements of nonlinear dynamics, stochastic processes and complexity research have permeated many fields of applied science but that even today fundamental questions are still under investigation. A compilation of recent advances is indeed timely. However, since this edition is also a Festschrift on the occasion of Ulrike Feudel's 60th birthday we should not conceal that the circle of contributors to this edition also reflects long-term collaborators and scientific disciples of Ulrike Feudel. Her own contributions to the developing field of nonlinear science and its applications, especially in ecological research, are manifold and widely recognized an appreciation of this is found in the opener of this edition [4] - and are also reflected by the fact that many contributors to this issue directly reference her results. Almost everybody that we, the editors, asked happily agreed to share an article for this edition. The responsibility for inviting authors is completely on the side of editors and we apologize to all those scientific colleagues that we missed and that deserved to be asked. We hope that this edition may serve the science community as a reference for the next few years and we dedicate it to Ulrike Feudel.

Happy Birthday, Ulrike!

\section{References}

1. E.N. Lorenz, J. Atmos. Sci. 20, 130 (1963)

2. T.Y. Li, J.A. Yorke, Am. Math. Mon. 82, 985 (1975)

3. R. Badii, A. Politi, Complexity: Hierarchical Structures and Scaling in Physics (Cambridge University Press, Cambridge, UK, 1999)

4. Y.-C. Lai, C. Grebogi, Eur. Phys. J. Special Topics 226, 1703 (2017)

5. P. Glendinning, Eur. Phys. J. Special Topics 226, 1721 (2017)

6. J. Rankin, H.M. Osinga, Eur. Phys. J. Special Topics 226, 1739 (2017)

7. Y. Saiki, E. Sander, J.A. Yorke, Eur. Phys. J. Special Topics 226, 1751 (2017)

8. V.M. Doroshenko, S.P. Kuznetsov, Eur. Phys. J. Special Topics 226, 1765 (2017)

9. E.V. Felk, A.V. Savin, A.P. Kuznetsov, Eur. Phys. J. Special Topics 226, 1777 (2017)

10. T. Arecchi, S. Euzzor, M.R. Gallas, J.A.C. Gallas, R. Meucci, E. Pugliese, S. Zambrano, Eur. Phys. J. Special Topics 226, 1785 (2017)

11. A. Politi, A. Pikovsky, E. Ullner, Eur. Phys. J. Special Topics 226, 1791 (2017)

12. E.H. Hellen, J. Kurths, S.K. Dana, Eur. Phys. J. Special Topics 226, 1811 (2017)

13. N. Rubido, C. Grebogi, M.S. Baptista, Eur. Phys. J. Special Topics 226, 1829 (2017)

14. F. Brenner, N. Marwan, P. Hoffmann, Eur. Phys. J. Special Topics 226, 1845 (2017)

15. E. Rybalova, N. Semenova, G. Strelkova, V. Anishchenko, Eur. Phys. J. Special Topics 226, 1857 (2017)

16. V. Maistrenko, O. Sudakov, O. Osiv, Y. Maistrenko, Eur. Phys. J. Special Topics 226, 1867 (2017)

17. J. Sawicki, I. Omelchenko, A. Zakharova, E. Schöll, Eur. Phys. J. Special Topics 226, 1883 (2017)

18. R. Banerjee, B.K. Bera, D. Ghosh, S.K. Dana, Eur. Phys. J. Special Topics 226, 1893 (2017)

19. A. Sharma, M.D. Shrimali, A. Prasad, R. Ramaswamy, Eur. Phys. J. Special Topics 226, 1903 (2017) 
20. J.M. Sausedo-Solorio, A.N. Pisarchik, Eur. Phys. J. Special Topics 226, 1911 (2017)

21. D.S. Goldobin, A.V. Pimenova, M. Rosenblum, A. Pikovsky, Eur. Phys. J. Special Topics 226, 1921 (2017)

22. A. Shaffer, R. Follmann, A.L. Harris, S. Postnova, H. Braun, E. Rosa Jr, Eur. Phys. J. Special Topics 226, 1939 (2017)

23. M. Khamesian, A.B. Neiman, Eur. Phys. J. Special Topics 226, 1953 (2017)

24. T. Rings, G. Ansmann, K. Lehnertz, Eur. Phys. J. Special Topics 226, 1963 (2017)

25. N. Martinez Alvarez, S. Borkar, C. Masoller, Eur. Phys. J. Special Topics 226, 1971 (2017)

26. J.C. Sprott, S.Jafari, A.J.M. Khalaf, T. Kapitaniak, Eur. Phys. J. Special Topics 226, 1979 (2017)

27. J.G. Freire, M.R. Gallas, J.A.C. Gallas, Eur. Phys. J. Special Topics 226, 1987 (2017)

28. F.G. Cosi, K. Krischer, Eur. Phys. J. Special Topics 226, 1997 (2017)

29. W. Ebeling, R. Feistel, Eur. Phys. J. Special Topics 226, 2015 (2017)

30. G. Drótos, T. Bódai, T. Tél, Eur. Phys. J. Special Topics 226, 2031 (2017)

31. S. Milster, J. Nötel, I.M. Sokolov, L. Schimansky-Geier, Eur. Phys. J. Special Topics 226, 2039 (2017)

32. E. Ser-Giacomi, V. Rodríguez-Méndez, C. López, E. Hernández-García, Eur. Phys. J. Special Topics 226, 2057 (2017)

33. K. Guseva, A. Daitche, T. Tél, Eur. Phys. J. Special Topics 226, 2069 (2017)

34. R.D. Vilela, V.M. de Oliveira, Eur. Phys. J. Special Topics 226, 2079 (2017)

35. S. Dan, M. Ghosh, Y. Nandukumar, S.K. Dana, P. Pal, Eur. Phys. J. Special Topics 226, 2089 (2017)

36. G. van Voorn, B.W. Kooi, Eur. Phys. J. Special Topics 226, 2101 (2017)

37. S. Chakraborty, P.K. Tiwari, S.K. Sasmal, A.K. Misra, J. Chattopadhyay, Eur. Phys. J. Special Topics 226, 2119 (2017)

38. R. Karnatak, S. Wollrab, Eur. Phys. J. Special Topics 226, 2135 (2017)

39. R. Arumugam, T. Banerjee, P.S. Dutta, Eur. Phys. J. Special Topics 226, 2145 (2017)

40. I. Siekmann, M. Bengfort, H. Malchow, Eur. Phys. J. Special Topics 226, 2157 (2017)

41. Y.R. Zelnik, O. Tzuk, Eur. Phys. J. Special Topics 226, 2171 (2017) 